\title{
Obesity leads to declines in motor skills across childhood
}

\author{
J. Cheng, ${ }^{\star}$ P. East, ${ }^{\star}$ E. Blanco, $\dagger$ E. Kang Sim, ${ }^{\star}$ M. Castillo, $\dagger$ B. Lozoff $\neq$ and S. Gahagan ${ }^{\star}$ \\ *University of California, San Diego, Department of Pediatrics, San Diego, CA, USA \\ $\dagger$ University of Chile, Institute of Nutrition and Food Technology, Santiago, Chile, and \\ $\ddagger$ University of Michigan, Center for Human Growth and Development, Ann Arbor, MI, USA
}

Accepted for publication 15 February 2016

\section{Keywords}

body mass index, motor skills, obesity, overweight status

Correspondence: Patricia East, Ph.D., University of California, San Diego, Department of Pediatrics, Center for Community Health, 4305 University Avenue, Suite 590, San Diego, CA 92105, USA E-mail: peast@ucsd.edu

\begin{abstract}
Background Poor motor skills have been consistently linked with a higher body weight in childhood, but the causal direction of this association is not fully understood. This study investigated the temporal ordering between children's motor skills and weight status at 5 and 10 years.

Methods Participants were 668 children (54\% male) who were studied from infancy as part of an iron deficiency anaemia preventive trial and follow-up study in Santiago, Chile. All were healthy, fullterm and weighing $3 \mathrm{~kg}$ or more at birth. Cross-lagged panel modelling was conducted to understand the temporal precedence between children's weight status and motor proficiency. Analyses also examined differences in gross and fine motor skills among healthy weight, overweight, and obese children.

Results A higher BMI at 5 years contributed to declines in motor proficiency from 5 to 10 years. There was no support for the reverse, that is, poor motor skills at 5 years did not predict increases in relative weight from 5 to 10 years. Obesity at 5 years also predicted declines in motor proficiency. When compared with normal weight children, obese children had significantly poorer total and gross motor skills at both 5 and 10 years. Overweight children had poorer total and gross motor skills at 10 years only. The differences in total and gross motor skills among normal weight, overweight and obese children appear to increase with age. There were small differences in fine motor skill between obese and non-obese children at 5 years only.

Conclusions Obesity preceded declines in motor skills and not the reverse. Study findings suggest that early childhood obesity intervention efforts might help prevent declines in motor proficiency that, in turn, may positively impact children's physical activity and overall fitness levels.
\end{abstract}

\section{Introduction}

Poor motor skills have been linked with higher body weight in childhood in a number of studies (Wrotniak et al. 2006; D'Hondt et al. 2011; Lopes et al. 2013). Overweight and obese children show poorer gross motor skill performance as well as worse fine motor precision and manual dexterity than their healthy weight peers (D'Hondt et al. 2009; Gentier et al. 2013).
To date, however, the temporal precedence, or causal direction, of this association is unclear. That is, it is currently not known whether high body mass contributes to poor motor skills or whether poor motor proficiency leads to higher body mass. Several longitudinal studies have been conducted in which only weight or motor skill have been used to predict subsequent changes in either weight or motor skill. For example, D'Hondt et al. (2013) reported that an unhealthy body mass index (BMI) 
at 6 to 10 years predicted declines in gross motor coordination performance 2 years later (D'Hondt et al. 2013). However, the reverse was not tested, that is, whether initial poor motor skills contribute to a subsequent unhealthy weight. Similarly, other studies have found that low levels of fitness and motor competence are strongly associated with the risk of subsequently becoming overweight or obese (Kim et al. 2005; Hruby et al. 2012; Rodrigues et al. 2016). However, these studies did not also test the possibility that being overweight or obese predicts subsequent declines in motor competence. Thus, from the available studies, the temporal ordering between children's motor skills and their body weight remains unclear. Understanding the causal ordering of the association between children's motor skill and their weight would be helpful for preventive purposes. In addition, examining this issue in early development might serve to identify the origins of the motor skill-adiposity link. That is, examining the within- and acrosstime associations between motor skill and adiposity starting at this stage in development might yield insights into uncovering the initiating factor.

\section{The current study}

The current study analyzed BMI and fine and gross motor skill data for 668 children who were studied at 5 and 10 years of age. Cross-lagged panel models (Selig \& Little 2012) were used to identify the temporal precedence in the relationship between children's motor skills and their weight status. Specifically, we analyzed how children's 5-year total motor skills (both gross and fine) predict changes in weight status from 5 to 10 years while simultaneously examining how 5-year weight status predicts changes in motor skills from 5 to 10 years. We used three indices of weight status: BMI $z$-scores, whether obese or not and whether overweight or not (omitting children who were obese at either time point). This approach allowed us to consider whether being overweight but not obese hinders children's motor skill development, as well as vice versa, that is, whether poor motor proficiency is a risk for subsequently becoming overweight for those who have not developed obesity. For these across-age analyses, total motor skills were examined or those that simultaneously consider both gross and fine motor skills. This is important to note because several studies in this field have examined age-related changes in gross motor skills only (e.g. D’Hondt et al. 2011, 2013).

In addition, this study examined whether healthy weight, overweight and obese children differ in their fine and gross motor skills at 5 and 10 years of age. Many previous studies that examined children's motor skill by weight status have compared only healthy weight and obese children (Gentier et al. 2013). Previous research also shows that weight class differences in gross motor coordination appear more pronounced in older (10-12 years) than younger (5-7 years) children (D'Hondt et al. 2011) and become more marked over time (D'Hondt et al. 2013; Lopes et al. 2013). The current study aims to clarify whether fine motor skills differ by children's weight status, will examine overweight children separately from obese children and will examine whether differences in children's motor skill among healthy weight, overweight and obese children become more marked over time.

\section{Methods}

\section{Participants}

Participants were 668 children (54\% male) who were studied as part of an iron deficiency anaemia preventive trial and follow-up study in Santiago, Chile (Lozoff et al. 2003). The study originally involved 1657 infants who were recruited from community clinics in Santiago between 1991 and 1996. All infants were healthy, full-term (birth weight $>3.0 \mathrm{~kg}$ ) and had no perinatal complications or acute or chronic illnesses. At infancy, children's families were working class, mothers had an average of 9-yeareducation (equivalent to ninth-gradeeducation) and $85 \%$ of fathers were present in the home. BMI and motor skills were measured at 5 and 10 years. Six hundred and sixty-eight children had complete BMI and motor proficiency data at both ages; these individuals form the sample for this study's analyses. The age periods of 5 and 10 years encompass important milestones in children's motor, cognitive and socioemotional development, including the refinement of perceptual and motor skills (Williams \& Stith 1980; Collins 1984). The infant study and the 5- and 10-year follow-ups were approved by the institutional review boards of the three participating universities. Signed informed consent was obtained from parents at all study time points, and assent was obtained from children at 10 years.

\section{Measures}

Motor skills

Children's motor skills were measured by the BruininksOseretsky Test of Motor Proficiency Short Form (BOTMP-SF; Bruininks 1978). The BOTMP-SF is a well-established, valid and reliable test of motor proficiency (Spironello et al. 2010). The BOTMP-SF includes 14 subtests, 6 of which assess gross 
motor skills (GM; e.g. running speed, balance, bilateral coordination), 6 that assess fine motor skills (FM; e.g. visualmotor control) and 2 that assess simultaneous gross and fine motor skills (e.g. grabs ball with both hands). A total standard score, adjusted for child age, is used to interpret motor skill proficiency. Thus, the total score reflects proficiency of gross and fine motor skills equally. The test was administered by five trained psychologists at the Institute of Nutrition and Food Technology (INTA), University of Chile. Inter-rater agreement of $>80 \%$ was established prior to the start of the study.

\section{Body mass index}

At 5 and 10 years, a research nurse at INTA, University of Chile measured children's height and weight. Weight was measured to $0.1 \mathrm{~kg}$ using a SECA scale. Height was measured to $0.1 \mathrm{~cm}$ using a Holtain stadiometer. BMI $\left(\mathrm{kg} / \mathrm{m}^{2}\right)$ was calculated and converted to $z$-scores using the methodology recommended by the Centers for Disease Control and Prevention (CDC) (Kuczmarski et al. 2002). BMI $z$-scores incorporate age and gender differences and thereby allow for comparisons across age and gender.

\section{Weight status}

Children's weight status was defined using CDC standards, with obesity defined as $>95$ th percentile and overweight as $>85$ th for age and gender (Kuczmarski et al. 2002).

\section{Covariates}

Based on correlational results (described in the succeeding texts), analyses controlled for child gender, the level of mothers'education when the child was 10 years old and the nurturing quality of the home environment at age 5 (measured using the Home Observation for Measurement of the Environment; possible score range: 0-45; Bradley et al. 1994). Iron deficiency and iron deficiency anaemia during infancy (scored as present or absent based on venous blood samples at 6,12 or 18 months) were also considered as covariates based on research that shows that iron-deficient anaemic infants are uncharacteristically lethargic, less physically active and have low energy (Lozoff 2007). However, neither iron deficiency anaemia nor iron deficiency in infancy was significantly associated with any of the model variables and was not considered further. Children's ages at the 5- and 10- year assessments (coded in months) were also not related to any of the study variables and were not considered further.

\section{Statistical analysis}

To examine initial relations among the study variables and to identify potential covariates, we conducted Pearson correlations. Next, we conducted analyses of covariance to determine whether healthy weight, overweight and obese children differ in their total, gross and fine motor skills at ages 5 and 10. These analyses controlled for child gender, 5-year Home Observation for Measurement of the Environment (HOME) scores and 10-year maternal education.

We then conducted cross-lagged panel analysis within a structural equation modelling framework (Selig \& Little 2012) to estimate the within- and across-time relations between children's weight and motor proficiency (using Mplus version 6.12; Muthén \& Muthén 1998-2007). Three models were analyzed. We examined the relations between children's motor proficiency and their (i) BMI; (ii) obesity status (yes, no); and (iii) overweight status (yes, no). As shown in Fig. 1, all models included autoregressive paths, within-time paths and across-time paths. Here, the autoregressive paths function as a control for the crosslagged path. Thus, a significant cross-lagged path when autoregressive paths are included can be interpreted as variable $e_{1}$ at time 1 is associated with change in variable from time 1 to time 2. Four model fit indices were used to examine how well the models fit the data: the chi-square, which is not significant when model fit is good (Bentler 1990); the comparative fit index, with

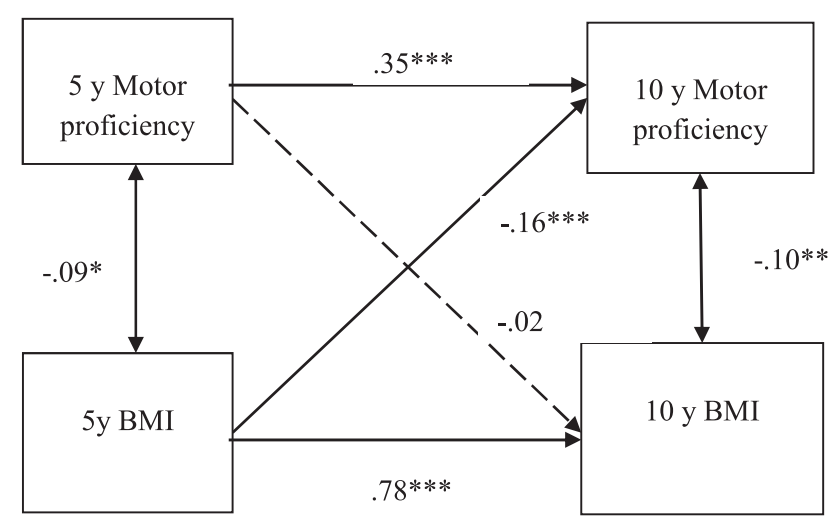

Figure 1. Cross-lagged panel model results for children's total motor proficiency score and BMI at 5 and 10 years, $n=668$. Standardized coefficients are shown. Dashed paths are not statistically significant. The model controlled for child gender, 5-year maternal education and 10-year home environment, not shown. Model fit indices were as follows: $\chi^{2}(4)$ $=5.24, n s$; CFI: 0.998; RMSEA: 0.022; SRMR: 0.014. ${ }^{\star} P<0.05,{ }^{*} P<0.01$, ${ }^{* * *} P<0.001$. 
Table 1. Descriptive characteristics of the sample $(n=668)$

\begin{tabular}{lcc}
\hline & $\mathbf{5}$ years & 10 years \\
\hline$\%$ Male & $54.0 \%$ & $54.0 \%$ \\
Maternal education (yrs) & $9.8(2.57)$ & $9.8(2.60)$ \\
HOME score* & $35.0(6.40)$ & $36.6(7.00)$ \\
Motor proficiency* a & $52.4(8.18)$ & $45.0(10.23)$ \\
BMl z-score* & $0.84(1.00)$ & $0.73(0.95)$ \\
$\%$ Overweight* & $20.4 \%$ & $22.9 \%$ \\
$\%$ Obese* & $21.7 \%$ & $18.1 \%$ \\
$\%$ Healthy weight & $57.9 \%$ & $59 \%$
\end{tabular}

Values given are percentages or means (SD).

a Summed standardized Bruininks-Oseretsky score reflecting total motor proficiency.

${ }^{*} P<0.001$, significant across-time change.

values above 0.90 indicating a reasonably good fit $(\mathrm{Hu} \&$ Bentler 1999); the root mean square error of approximation, with values less than 0.08 indicating adequate fit (Kline 2011); and the standardized root mean square residual, which is a measure of the mean absolute value of the covariance residuals, with values less than 0.08 considered favourable (Bentler 1990).

\section{Results}

At age 5, 20.4\% of children were overweight and $21.7 \%$ were obese (combined 42.1\%; Table 1). At age 10, 22.9\% were overweight and $18.1 \%$ were obese (combined $41.0 \%$ ). There were no gender differences in these weight classifications. Table 1 also shows mean motor proficiency scores and BMI $z$ scores at 5 and 10 years. Both BMI $z$-scores $(t[667]=4.15$, $P<0.001)$ and motor proficiency scores $(t[667]=18.74$, $P<0.001)$ decreased across age.

Pearson correlations (Table 2) indicated that BMI $z$-scores and motor proficiency scores were significantly negatively correlated within-time at both age points. Five-year BMI $z$ - scores and motor proficiency were also strongly and significantly correlated with their respective values at 10 years. Gender was correlated with motor proficiency at both ages, such that boys had higher total motor scores than girls. In addition, children's nurturing home environment at 5 years was positively correlated with their 10 -year motor proficiency, and 10-year maternal education was positively correlated with children's 10-year BMI. Given these relations, gender was included as a covariate on 5- and 10-year motor proficiency scores in the cross-lagged modelling analyses; the 5-year HOME score was included as a covariate on 10-year motor proficiency; and mothers' education at 10 years was included as a covariate on children's 10-year BMI $z$-score.

\section{Weight group differences}

The mean total, gross and fine motor skill scores for healthy weight, overweight and obese children at ages 5 and 10 are shown in Table 3. Results of the ANCOVA and post hoc contrasts indicate that, at 5 years, obese children had poorer total and gross motor skills than both their healthy weight and overweight peers. At age 10, both obese and overweight children differed from healthy weight children in total and gross motor proficiency. Also at age 10, obese children had lower gross motor proficiency scores than overweight children. Figure 2 graphically illustrates the total mean skill values per weight group at both ages. The differences in total and gross motor skills among normal weight, overweight and obese children appear to increase with age.

There were no differences among the groups in fine motor skills at either age. However, post hoc analyses of the individual fine motor subtests showed one significant difference: Obese children performed worse on a fine motor precision task at 5 years (drawing lines) than both overweight and healthy

Table 2. Correlations among model variables and covariates $(n=668)$

5-year motor proficiency 5-year BMI z-score 5-year obesity 10-year motor proficiency 10-year BMI z-score 10-year obesity

\begin{tabular}{|c|c|c|c|c|c|c|}
\hline 5-year motor proficiency & - & & & & & \\
\hline 5-year BMI z-score & $-0.09^{*}$ & - & & & & \\
\hline 5-year obesity ${ }^{a}$ & $-0.16^{* * *}$ & $0.62^{* * *}$ & - & & & \\
\hline 10-year motor proficiency & $0.40^{* * *}$ & $-0.19^{* * *}$ & $-0.16^{* * *}$ & - & & \\
\hline 10-year BMI z-score & $-0.09^{*}$ & $0.78^{* * *}$ & $0.47^{* * *}$ & $-0.19^{* * *}$ & - & \\
\hline 10-year obesity ${ }^{a}$ & $-0.12^{* *}$ & $0.48^{* * *}$ & $0.61^{* * *}$ & $-0.15^{* * *}$ & $0.44^{* * *}$ & - \\
\hline Child gender ${ }^{\mathrm{b}}$ & $0.13^{* * *}$ & -0.03 & 0.07 & $0.26^{* * *}$ & 0.05 & 0.07 \\
\hline 5-year mothers' education & 0.00 & 0.04 & -0.01 & 0.02 & 0.07 & 0.02 \\
\hline 5-year HOME & 0.07 & 0.05 & 0.03 & $0.10^{* *}$ & 0.06 & 0.01 \\
\hline 10-year mothers' education & -0.03 & 0.01 & -0.02 & 0.06 & $0.08^{*}$ & 0.01 \\
\hline 10-year HOME & 0.06 & 0.07 & 0.06 & 0.06 & 0.05 & 0.03 \\
\hline
\end{tabular}

${ }^{\mathrm{a}} 0=$ no, $1=$ yes. $^{\mathrm{b}} 0=$ female, $1=$ male.

${ }^{*} P<0.05 .{ }^{* *} P<0.01 .{ }^{* * *} P<0.001$. 
Table 3. Total, gross and fine motor skills at 5 and 10 years, stratified by healthy weight (HW), overweight (OW) and obesity (OB) status

\begin{tabular}{|c|c|c|c|c|c|}
\hline & HW & ow & OB & $F[2,666]$ & Contrast \\
\hline 5 years $(n=668)$ & 387 & 136 & 145 & & \\
\hline Total motor proficiency standardized score & $53.0(8.1)$ & $52.8(7.5)$ & $50.3(8.6)$ & $6.62^{*}$ & $\mathrm{HW}>\mathrm{OB} ; \mathrm{OW}>\mathrm{OB}$ \\
\hline Gross motor skills & $13.7(3.8)$ & $13.3(3.9)$ & $11.9(4.3)$ & $11.08^{* *}$ & $\mathrm{HW}>\mathrm{OB} ; \mathrm{OW}>\mathrm{OB}$ \\
\hline Fine motor skills & $13.3(2.9)$ & $13.4(2.8)$ & $13.1(3.1)$ & $<1$ & \\
\hline 10 years $(n=668)$ & 394 & 153 & 121 & & \\
\hline Total motor proficiency standardized score & $45.8(10.1)$ & $43.5(10.1)$ & $40.9(9.5)$ & $24.09^{* *}$ & $\mathrm{HW}>\mathrm{OW} ; \mathrm{HW}>\mathrm{OB}$ \\
\hline Gross motor skills & $25.4(3.9)$ & $23.8(4.0)$ & $21.9(4.1)$ & $47.06^{* *}$ & $\mathrm{HW}>\mathrm{OW}$; HW $>\mathrm{OB} ; \mathrm{OW}>\mathrm{OB}$ \\
\hline Fine motor skills & 26.9 (3.6) & $26.5(3.8)$ & $26.5(3.6)$ & 1.49 & \\
\hline
\end{tabular}

Values are means (SD). $F$ values controlled for child gender, 5-year HOME scores and 10-year maternal education.

${ }^{*} P<0.01$. ${ }^{*} P<0.001$

weight children $(F[2,666]=3.00, P<0.05$, controlling for child gender). There were no group differences on any of the fine motor subtests at age 10 .

\section{Modelling results}

The model fit indices for the crossed-lagged panel model analyzing children's motor skills and BMI indicated good model fit (Fig. 1). At both time points (and consistent with the correlations), children's motor skills and BMI were significantly negatively associated. The cross-lagged paths indicated that a higher BMI at 5 years was associated with significant declines in motor skills from 5 to 10 years $(\beta=-0.16$, $P<0.001)$. However, the reverse was not true, that is, 5-year motor proficiency was not related to changes in relative weight from 5 to 10 years $(\beta=-0.02, n s)$.

Figure 3 shows the cross-lagged panel model of children's motor skills and their obesity status (yes, no). Model fit indices again indicated that model fit was good. These results were

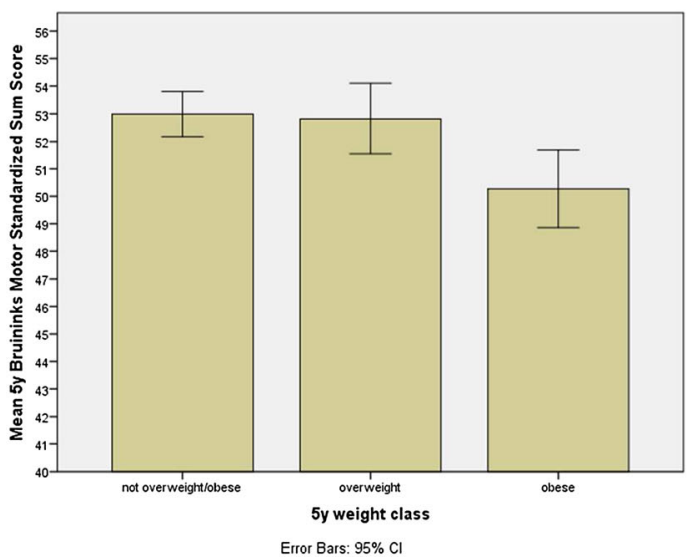

similar to those using BMI $z$-scores, such that an obese status at age 5 was associated with significant declines in motor proficiency from age 5 to $10(\beta=-0.12, P<0.01)$, but 5 -year motor skills were unrelated to change in obesity status from 5 to 10 years $(\beta=-0.03, n s)$.

Figure 4 shows the cross-lagged model analyzing overweight status (yes, no; and omits those who were obese at either time point, or 182 children). The model fit indices indicated that model fit was good. In this model, an overweight status at age 5 was marginally associated with declines in motor proficiency from age 5 to $10(\beta=-0.07, P<0.09)$, while 5 -year motor skills were unrelated to a change in overweight status from 5 to 10 years $(\beta=-0.06, n s)$.

\section{Discussion}

We found that a high BMI and, separately, being obese at age 5 predicted declines in children's motor skills from 5 to 10 years. The reverse was not found, that is, poor motor proficiency at

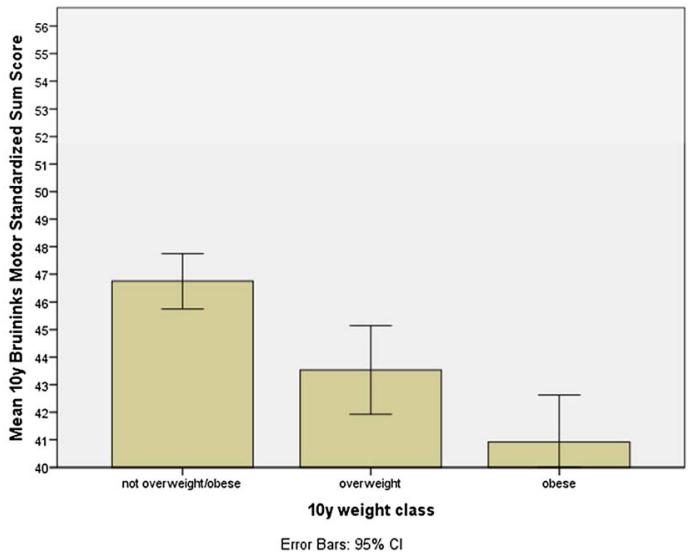

Figure 2. Mean total standardized motor skills per weight group at age 5 years (left) and 10 years (right). 


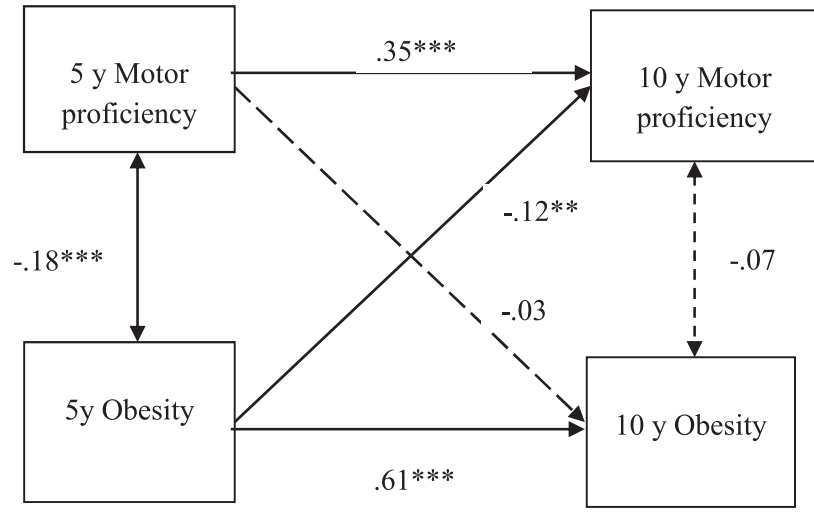

Figure 3. Cross-lagged panel model results for children's total motor proficiency score and obesity status (yes, no; coded 1,0 ) at 5 and 10 years, $n=668$. Standardized coefficients are shown. Dashed paths are not statistically significant. The model controlled for child gender, 5-year maternal education and 10-year home environment, not shown. Model fit indices were as follows: $\chi^{2}(3)=4.64, n s$; CFI: 0.996; RMSEA: 0.029; SRMR: 0.017. ${ }^{*} P<0.01,{ }^{* * *} P<0.001$.

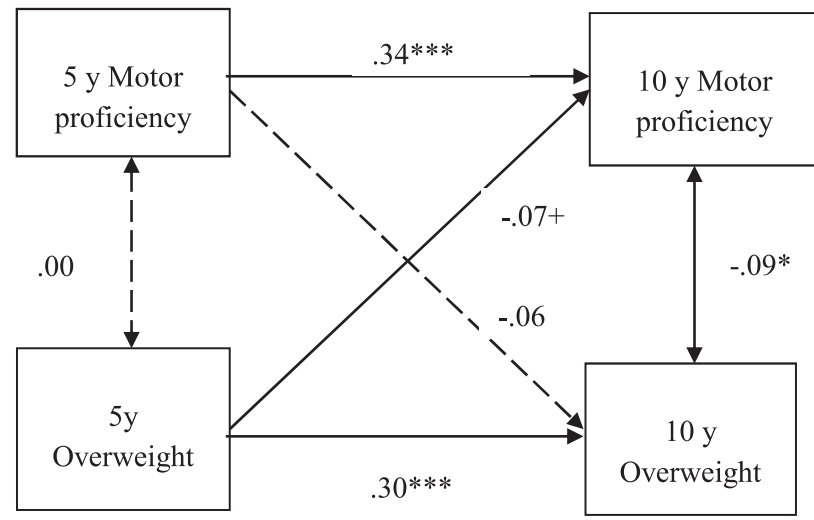

Figure 4. Cross-lagged panel model results for children's total motor proficiency score and overweight status (yes, no; coded 1, 0; excludes obese children) at 5 and 10 years, $n=486$. Standardized coefficients are shown. Dashed paths are not statistically significant. The model controlled for child gender, 5-year maternal education and 10-year home environment, not shown. Model fit indices were as follows: $\chi^{2}(3)=5.50, n s$; CFI: 0.985; RMSEA: 0.041; SRMR: 0.019. $+P<0.09,{ }^{*} P<0.05,{ }^{* * *} P<0.001$.

age 5 did not predict obesity or increases in BMI. These findings indicate that a heavier weight status is a precursor and not a consequence of poor motor skills. Results also show that being overweight at age 5 was not sufficient to produce significant declines in motor skills from 5 to 10 years. However, marginally significant declines in motor skill were associated with overweight status at age 5, which might hold future significance, particularly if these children become obese at a later age. The overall pattern of results suggests that excess adiposity might inhibit obese children from developing and exercising their motor skills, which contributes to declines in motor proficiency relative to healthy weight children (Castetbon \& Andreyeva 2012). These findings are clinically important because poor motor skill development is associated with children's physical inactivity and poor fitness levels (Wrotniak et al. 2006; Burgi et al. 2011). Relative declines in children's motor proficiencies might also be associated with self-perceptions of poorer physical competence, which again might serve as a catalyst for inactivity and consequent weight gain (Spessato et al. 2012).

We also observed significant differences in total and gross motor proficiency depending on children's weight status at both 5 and 10 years. When compared with normal weight children, obese children had significantly poorer total and gross motor skills at both ages. Overweight children exhibited poorer total and gross motor skills than normal weight children at 10 years only. In addition, overweight children had better total motor proficiency than obese children at 5 years but by age 10 were equivalent to obese children in total motor proficiency. Thus, overweight children appear to be performing more like obese children in terms of motor skills at age 10. The differences in total and gross motor skills among healthy weight, overweight and obese children appeared to increase with age. This is consistent with previous research showing that weight class differences in gross motor coordination become more pronounced at older ages (D'Hondt et al. 2011, 2013). These findings suggest the need to address children's weight issues at an early age, prior to the onset of large disparities in motor skills between healthy weight and unhealthy weight children.

Some prior studies have shown fine motor skill impairments in obese children (D'Hondt et al. 2009; Gentier et al. 2013). In our study, there were no group differences in children's overall fine motor skills at either time point, but obese children performed worse than overweight and healthy weight children on a line drawing task at 5 years. This result suggests that fine motor impairments may be evident in obese children, although confined to a specific fine motor ability and not persistent. Further investigation to corroborate this finding is warranted.

\section{Limitations and strengths}

The current study was unable to compute cross-lagged analyses for gross and fine motor skills separately because these subtest scores of the BOTMP-SF are not standardized and thus could not be compared across age; only the BOTMP-SF total motor proficiency scores could be analyzed across age. Nevertheless, the ANCOVA results provide valuable information indicating 
that the weight groups differ primarily in gross motor skill at both ages 5 and 10. We also could not compare mean level motor score differences by weight group across age because weight group status changed for $32 \%$ of participants between 5 and 10 years. In addition, to address the possible influence of children's socioeconomic background on the relations between motor skills and weight status, we controlled for mothers' years of education as a proxy for socioeconomic status (Krieger et al. 1997). Thus, the results found adjusted for children's socioeconomic background. It is possible, however, that unmeasured features in children's environments could have influenced the relations found. For example, children from more enriched environments could have more opportunity for motor skill development than children from poorer environments (Estabrooks et al. 2003), which could influence children's weight status. Socioeconomic factors and their association with children's weight and motor skill development should be considered more specifically in research of this kind. Finally, because this study involved Chilean children, it is not known whether the current findings would hold in a population with different obesity rates, as well as different cultural norms for physical activity. The degree to which study results may differ for children of other cultures is not known.

Strengths of the study are that the cross-lagged models were tested using a large sample and included several relevant statistical controls. Thus, the relations that emerged did so while controlling for key child and family characteristics known to be correlated with both child weight (Wang 2001) and motor skill (Okely \& Booth 2004). In addition, the crosslagged models simultaneously tested both possible across-time relations between children's weight and motor skill, thereby providing an opportunity to discern temporal ordering between these measures and a likely causal direction of effects. Because 5-year measures of each construct were included in the model, we were able to assess the effects of 5-year motor skill and weight status on subsequent changes in the opposing measure. In addition, our analyses considered obese children separate from overweight children, and we included an analysis of differences in fine motor skills vis-à-vis children's weight status group.

\section{Conclusion}

The purpose of this study was to examine the temporal ordering of children's relative weight and motor skills, a question with considerable practical significance. The results indicate that a high relative weight contributes to subsequent declines in motor skills and not the reverse. These findings suggest that early childhood obesity intervention efforts might help prevent subsequent declines in children's motor proficiency, which may produce secondary benefits of positively impacting children's physical activity and fitness levels (Wrotniak et al. 2006; Rodrigues et al. 2016). The results also suggest that intervention efforts that target overweight and obese children might be best timed prior to kindergarten or first grade or before significant declines in motor skill occur.

\section{Key messages}

- A higher BMI and, separately, being obese at 5 years were associated with significant declines in motor proficiency from 5 to 10 years.

- Motor skills at 5 years were unrelated to changes in either BMI or weight status from 5 to 10 years.

- The differences in total and gross motor skills among healthy weight, overweight and obese children appear to increase with age.

- Early childhood obesity intervention efforts might help minimize subsequent declines in children's motor skills and serve to increase physical activity and overall fitness.

\section{Funding}

Grant R01-HL-088530 from the National Institute of Heart, Lung and Blood Institute (PI: S.G); Grant R01-HD33487 from the National Institute of Child Health and Human Development (PIs: Lozoff \& Gahagan)

\section{References}

Bentler, P. (1990) Comparative fit indices in structural models. Psychological Bulletin, 107, 238-246.

Bradley, R. H., Mundfrom, D. J., Whiteside, L., Casey, P. H. \& Barrett, K. (1994) A factor analytic study of the infant-toddler and early childhood versions of the HOME Inventory administered to white, Black, and Hispanic American parents of children born preterm. Child Development, 65, 880-888.

Bruininks, R. (1978) Bruininks-Oseretsky Test of Motor Proficiency. American Guidance Service, Circle Pines, MN.

Burgi, F., Meyer, U., Granacher, U., Schindler, C., Marques-Vidal, P., Kriemler, S. \& Puder, J. J. (2011) Relationship of physical activity with motor skills, aerobic fitness and body fat in preschool children: a cross-sectional and longitudinal study. International Journal of Obesity, 35, 937-944.

Castetbon, K. \& Andreyeva, T. (2012) Obesity and motor skills among 4 to 6-year-old children in the United States: nationallyrepresentative surveys. BMC Pediatrics, 12, 28-36. 
Collins, W. A. (ed.) (1984) Development during middle childhood: the years from six to twelve. National Academies Press, Washington, DC. D’Hondt, E., Deforche, B., De Bourdeaudhuij, I. \& Lenoir, M. (2009) Relationship between motor skill and body mass index in 5-to 10-yearold children. Adapted Physical Activity Quarterly, 26, 21-37.

D’Hondt, E., Deforche, B., Gentier, I., De Bourdeaudhuij, I., Vaeyens, R., Philippaerts, R. \& Lenoir, M. (2013) A longitudinal analysis of gross motor coordination in overweight and obese children versus normal-weight peers. International Journal of Obesity, 37, 61-67.

D'Hondt, E., Deforche, B., Vaeyens, R., Vandorpe, V., Vandendriessche, J., Pion, J., Philippaerts, R., De Bourdeaudhuij, I. \& Lenoir, M. (2011) Gross motor coordination in relation to weight status and age in 5-to 12-year-old boys and girls: a cross-sectional study. International Journal of Pediatric Obesity, 6, e556-e564.

Estabrooks, P. A., Lee, R. E. \& Gyurcsik, N. C. (2003) Resources for physical activity participation: does availability and accessibility differ by neighborhood socioeconomic status? Annals of Behavioral Medicine, 25, 100-104.

Gentier, I., D’Hondt, E., Shultz, S., Deforche, B., Augustijn, M., Hoorne, S., Verlaecke, K., De Bourdeaudjuij, I. \& Lenoir, M. (2013) Fine and gross motor skills differ between healthy-weight and obese children. Research on Developmental Disabilities, 34, 4043-4051.

Hruby, A., Chomitz, V. R., Arsenault, L. N., Must, A., Economos, C. D., McGowan, R. J. \& Sacheck, J. M. (2012) Predicting maintenance or achievement of healthy weight in children: the impact of changes in physical fitness. Obesity, 20, 1720-1717.

Hu, L. T. \& Bentler, P. M. (1999) Cutoff criteria for fit indexes in covariance structure analysis: conventional criteria versus new alternatives. Structural Equation Modeling, 6, 1-55.

Kim, J., Must, A., Fitzmaurice, G. M., Gillman, M. W., Chomitz, V., Kramer, E., McGowan, R. \& Peterson, K. E. (2005) Relationship of physical fitness to prevalence and incidence of overweight among school children. Obesity Research, 13, 1246-1254.

Kline, R. B. (2011) Principles and practice of structural equation modeling, 3rd edn. Guilford Press, New York, NY.

Krieger, N., Williams, D. R. \& Moss, N. (1997) Measuring social class in U.S. public health research: concepts, methodologies, and guidelines. Annual Review of Public Health, 18, 341-378.

Kuczmarski, R. J., Ogden, C. L., Guo, S. S., Grunner-Stawn, L. M., Flegal, K. M., Mei, Z., Wei, R., Curtin, L. R., Roche, A. F. \& Johnson,
C. L. (2002) 2000 CDC growth charts for the United States: methods and development. Vital Health Statistics, Series, 11, 1-190.

Lopes, V. P., Stodden, D. F. \& Rodrigues, L. P. (2013) Weight status is associated with cross-sectional trajectories of motor co-ordination across childhood. Child: Care, Health and Development, 40, 891-899.

Lozoff, B. (2007) Iron deficiency and child development. Food and Nutrition Bulletin, 28, S560-S571.

Lozoff, B., DeAndraca, I., Castillo, M., Walter, T., Smith, J. \& Pino, P. (2003) Behavioral and developmental effects of preventing irondeficiency anemia in healthy full-term infants. Pediatrics, 112, 846-854. Muthén, L. K. \& Muthén, B. O. (1998-2007) Mplus user's guide, 5th edn. Muthén \& Muthén, Los Angeles, CA.

Okely, A. \& Booth, M. (2004) Master of fundamental movement skills among children in New South Wales: prevalence and sociodemographic distribution. Journal of Science and Medicine in Sport, 7, 358-372.

Rodrigues, L. P., Stodden, D. F. \& Lopes, V. P. (2016) Developmental pathways of change in fitness and motor competence are related to overweight and obesity status at the end of primary school. Journal of Science and Medicine in Sport, 19, 87-92.

Selig, J. P. \& Little, T. D. (2012) Autoregressive and cross-lagged panel analysis for longitudinal data. In: Handbook of Developmental Research Methods (eds B. Laursen, T. D. Little \& N. A. Card), pp. 265-278. Guilford Press, New York.

Spessato, B. C., Gabbard, C., Robinson, L. \& Valentini, N. C. (2012) Body mass index, perceived and actual physical competence: the relationship among young children. Child: Care, Health and Development, 39, 845-850.

Spironello, C., Hay J., Missiuna, C., Faught, B. E. \& Cairney, J. (2010) Concurrent and construct validation of the short form of the Bruininks-Oseretsky Test of Motor Proficiency and the Movement$\mathrm{ABC}$ when administered under field conditions: implications for screening. Child: Care, Health and Development, 36, 499-507.

Wang, Y. (2001) Cross-national comparison of childhood obesity: the epidemic and the relationship between obesity and socioeconomic status. International Journal of Epidemiology, 30, 1129-1136.

Williams, J. \& Stith, M. M. (1980) Middle childhood: behavior and development. Macmillan Publishing Company, New York, NY, USA.

Wrotniak, B. H., Epstein, L. H., Dorn, J. M., Jones, K. E. \& Kondilis, V. A. (2006) The relationship between motor proficiency and physical activity in children. Pediatrics, 118, e1758-e1765. 